\title{
Dynamic Selection and Update of Digital Predistorter Coefficients for Power Amplifier Linearization
}

\author{
Quynh Anh Pham*, David López-Bueno \#*, Gabriel Montoro* and Pere L. Gilabert* \\ ${ }^{*}$ Dept. Signal Theory and Comm., Universitat Politècnica de Catalunya (UPC), Castelldefels, Spain \\ \#Centre Tecnològic de Telecomunicacions de Catalunya (CTTC/CERCA), Castelldefels, Spain
}

\begin{abstract}
In this paper, a new method for dynamically estimating and updating the coefficients of a digital predistortion (DPD) linearizer is presented. By means of the partial least squares (PLS) algorithm, the basis matrix used in the DPD estimation/adaptation is dynamically updated at every iteration to minimize the linearization error. Moreover, only the minimum necessary DPD coefficients being required to meet a target estimation error are computed. The proposed estimation technique is compared with the standard least squares (LS) estimation solved by using QR decomposition. Experimental results show the similar linearization performance obtained with both estimation methods, but in the case of the dynamic PLS, using less coefficients at every iteration. Finally, the proposed algorithm allows a high level of parallelization, which makes it suitable for FPGA implementation.

Index Terms-Digital predistortion, power amplifier linearization, partial least squares regression.
\end{abstract}

\section{INTRODUCTION}

Digital predistortion (DPD) is the most popular solution to cope with the nonlinear behavior of power amplifiers (PAs) in wireless communication systems. Some technologies arisen with $4 \mathrm{G}$ communications such as carrier aggregation or MIMO, continue to develop for $5 \mathrm{G}$, where the always growing signal bandwidths and peak to average power ratios (PAPRs) challenging the DPD linearizers when coping with the PAs' unwanted nonlinear distortion effects. In addition, to linearize high efficient amplification architectures based on dynamic bias supply or dynamic load modulation, the DPD behavioral model significantly grows in number of parameters. This not only increases the computational complexity, but also can lead to uncertainty of the LS estimation, when accounting for DPD linearization.

In order to deal with the overfitting and uncertainty of the LS estimation in the adaptation subsystem, many efforts have been made to reduce the number of necessary DPD basis functions. The DPD order reduction can be done by properly selecting the most relevant basis functions (e.g., using a greedy algorithm such as the orthogonal matching pursuit -OMP- [1]) or by creating a new set of components that are the linear combinations of the original basis (e.g. using principal component analysis PCA- [2] or partial least squares -PLS- [3]). Alternatively, both approaches, (i.e., OMP combined with PLS/PCA) can be properly combined as in [4]. In addition, as discussed in [4], the order reduction obtained without loss of performance when using PLS is higher than with PCA, since the PLS obtains the new transformed basis of components taking into account the information of the PA output [3].

Unlike in [4], where a new basis with a reduced but fixed number of properly selected components was obtained to guarantee a well-conditioned estimation, in this paper, PLS is employed inside the DPD adaptation loop to actively adjust the basis matrix in the DPD identification subsystem. The dynamic basis reduction is carried out at every iteration according to the residual linearization error, defined as the difference between the actual and the desired linear PA output signals. In comparison to the QR decomposition (commonly used in conventional DPD estimation/adaptation to avoid the costly mathematical computation of the least squares (LS) Moore-Penrose inverse of the covariance matrix), the proposed technique allows to dynamically adjust the number of coefficients to meet the targeted linearity level. As it will be proved in the following subsections, the proposed solution leads to a reduction of the number of estimated DPD coefficients (which impacts in the computational complexity reduction) and guarantees a well-conditioned and robust estimation.

\section{DPD ESTIMATION/ADAPTATION WITH DYNAMIC BASIS MATRIX}

In a closed-loop direct learning approach (see Fig. 1), the DPD coefficients are iteratively updated as follows,

$$
\mathbf{w}_{i+1}=\mathbf{w}_{i}+\mathbf{d w}
$$

with $\mathbf{w}_{i}$ being the $M \times 1$ vector of coefficients of the DPD model at the $i^{\text {th }}$ iteration. The LS estimation of the coefficients' increment $\mathbf{d w}$ is

$$
\mathbf{d w}=\mu\left(\mathbf{U}^{H} \mathbf{U}\right)^{-1} \mathbf{U}^{H} \mathbf{e}
$$

where $\mu$ is the learning-rate parameter. The linearization error is defined as $\mathbf{e}=\frac{\mathbf{y}}{G_{0}}-\mathbf{u}$, where $G_{0}$ is the desired PA linear gain, the $N \times 1$ vectors $\mathbf{y}$ and $\mathbf{u}$ are the PA output and input signals, respectively; and the $N \times M$ 


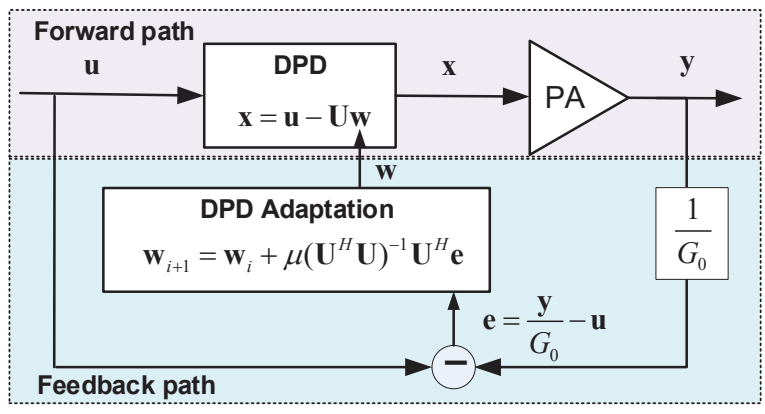

Fig. 1. An adaptive DPD system following a direct learning approach.

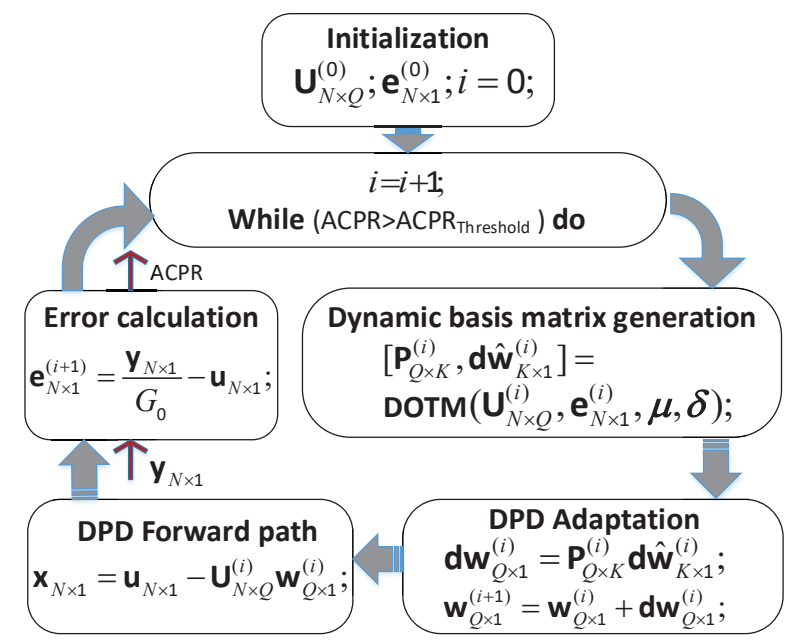

Fig. 2. Flowchart of the DPD estimation/adaptation using dynamic basis matrix.

data matrix $\mathbf{U}$ contains the $M$ basis functions describing the DPD behavioral model.

First, to reduce the number of coefficients in the DPD forward path, OMP is applied to choose the most relevant basis functions of $\mathbf{U}$. Then, to avoid calculating the MoorePenrose inverse of the covariance matrix, i.e., $\left(\mathbf{U}^{H} \mathbf{U}\right)^{-1}$, one of the most common solution is to extract the coefficients through QR decomposition combined with LS.

However, in this paper, the PLS regression [3] is implemented inside the DPD adaptation loop to dynamically adjust the basis matrix in the DPD subsystem. Fig. 2 depicts the proposed DPD estimation/adaptation employing dynamic basis matrix approach. Thanks to the proposed Dynamic Orthonormal Transformation Matrix (DOTM) algorithm, at every iteration we obtain a new transformed matrix with the minimum necessary number of new components. The DOTM algorithm is a modification of the iterative SIMPLS algorithm [5] for PLS regression, that calculates the linear combinations of the original basis with maximum covariance between these new basis and the signal to be estimated. Whereas the size

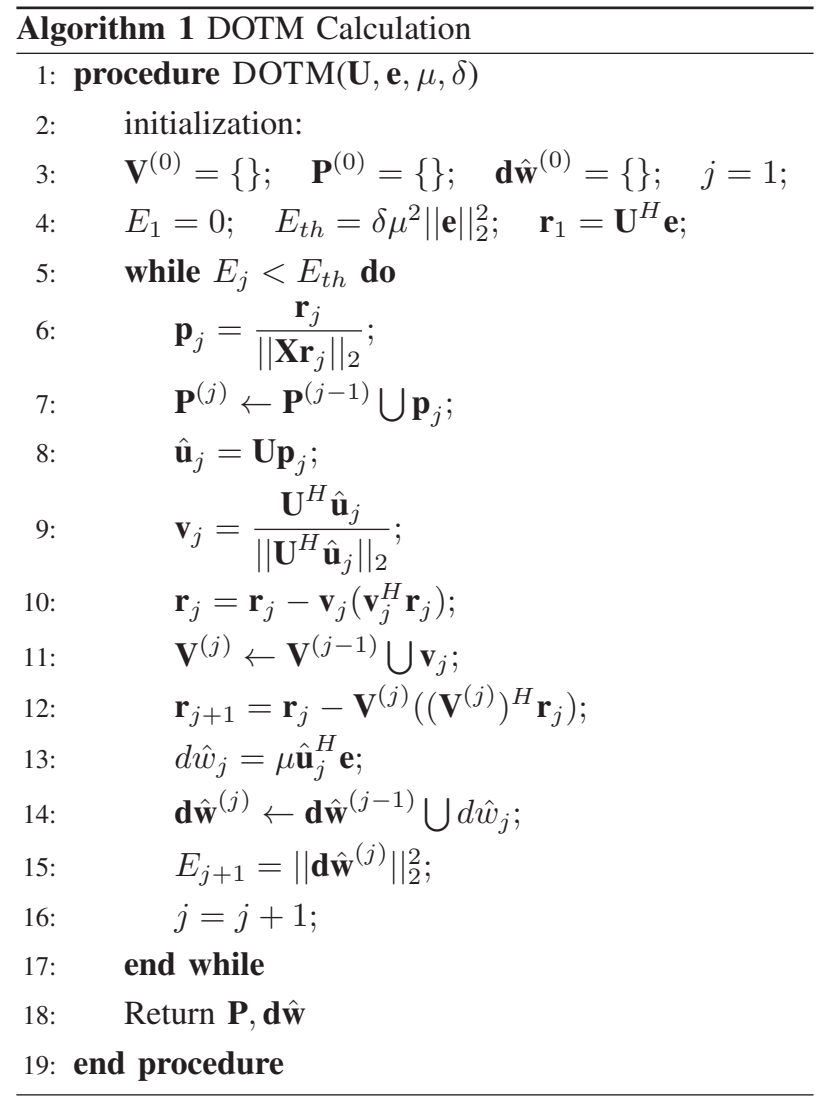

of the transformation matrix $\mathbf{P}$ is predetermined and given as an input information in SIMPLS, in DOTM the number of columns of $\mathrm{P}$ and coefficients are iteratively added and calculated until the power of the estimated error is close enough to the threshold $E_{t h}$, defined as a percentage $\delta$ of the power of the error signal e. By taking into account the orthonormality among the components, the power of the estimated error corresponds to the power of the estimated coefficients. Therefore, thanks to the DOTM algorithm, at each iteration $i$ of the DPD adaptation, the number of columns of the transformation matrix $\mathbf{P}$ varies, and only the minimum necessary number of columns that meet the $E_{t h}$ threshold requirements are selected. The $Q \times K$ transformation matrix $\mathbf{P}$ obtained from DOTM is used for computing the transformed basis matrix $\hat{\mathbf{U}}$,

$$
\hat{\mathbf{U}}=\mathbf{U P}
$$

with $\mathbf{U}$ being the $N \times Q$ matrix of basis functions after applying OMP. The new transformed matrix $\hat{\mathbf{U}}$ presents orthonormal components and has dimensions $N \times K$, where the number of components $K$ (with $K \leq Q$ ) is variable since depends on the dimensions of $\mathbf{P}$. The new orthonormal components (i.e. columns) of the transformed matrix $\hat{\mathbf{U}}$ are sorted according to their contribution to maximize the covariance between the new components and 


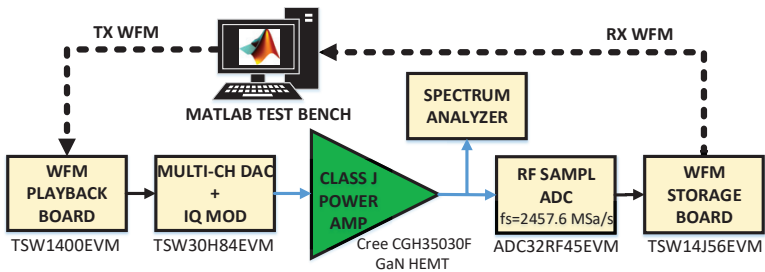

Fig. 3. High-level block diagram of the Matlab-controlled digital linearization test bench.

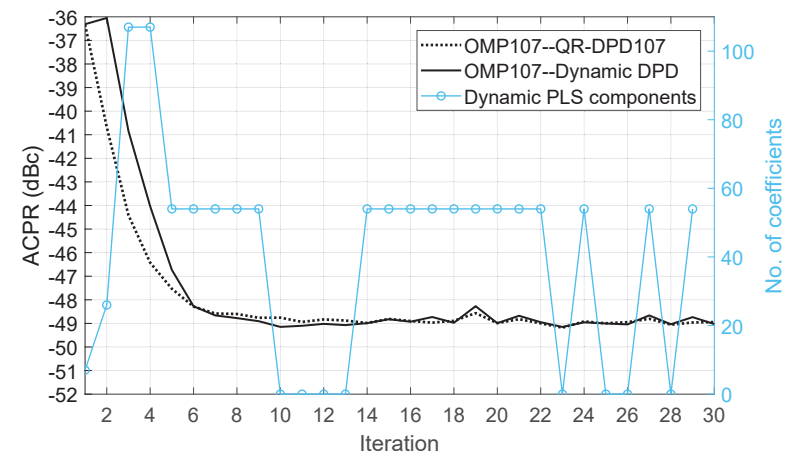

Fig. 4. The DPD estimation/adaptation with DOTM vs. with QR decomposition.

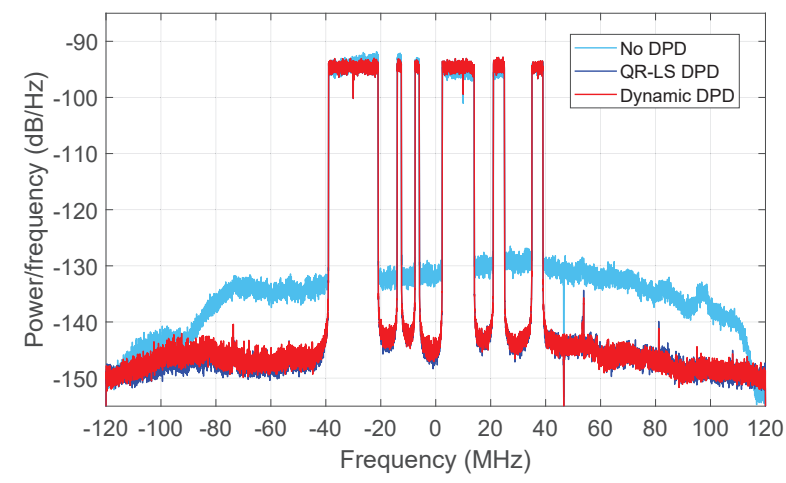

Fig. 5. Spectra of the PA output before and after dynamic DPD linearization.

the error signal e. Taking into account the orthonormal property of the transformed matrix $\hat{\mathbf{U}}$ (i.e., $\hat{\mathbf{U}}^{H} \hat{\mathbf{U}}=\mathbf{I}$ ), the update of the transformed coefficients is simplified,

$$
\mathbf{d} \hat{\mathbf{w}}=\mu \hat{\mathbf{U}}^{H} \mathbf{e}
$$

Finally, the original coefficients' increment is obtained through the following anti-transformation,

$$
\mathbf{d w}=\mathbf{P d} \hat{\mathbf{w}}
$$

As described in Fig. 2, the DPD adaptation will continue until achieving the desired ACPR level.

\section{EXPERIMENTAL RESULTS}

The dynamic selection and update of the DPD coefficients described in Fig. 2 was validated in a Matlab controlled hardware test bench as shown in Fig. 3. An $80 \mathrm{MHz}$ bandwidth carrier-aggregated fast convolution filter bank multi-carrier (FC-FBCM) signal with subcarrier group deactivation was up-converted to the $875 \mathrm{MHz} \mathrm{RF}$ frequency to feed a class-J PA.

The original basis functions were generated by using the generalized memory polynomial (GMP) behavioral model with 322 coefficients. By applying OMP, we reduced the number of coefficients up to 107. Then, we performed two tests: a) DPD estimation/adaptation with QR decomposition using 107 coefficients and $b$ ) DPD estimation/adaptation using the DOTM algorithm to generate dynamic basis matrix with an ACPR target of -48 dBc. As shown in Fig. 4, both DPD adaptation methods converge after 10 iterations to the same ACPR level. However, while the DPD adaptation with QR-LS requires a constant number of 107 coefficients at every iteration to converge, the DPD adaptation with the DOTM algorithm (that dynamically adjusts the dimensions of the basis matrix), needs less coefficients (only at iterations 3 and 4 it needs up to 107 coefficients) to achieve the same targeted ACPR value. Finally, Fig. 5 shows the unlinearized and linearized spectra considering the QR-LS and the proposed dynamic selection and update of the DPD coefficients. Despite showing similar performance, when comparing the measured running time for both algorithms implemented in Matlab plain code, the DOTM algorithm is around 50 times faster than the case of QR.

\section{CONCLUSION}

In this paper we have proposed a new method for dynamically estimating and updating the DPD coefficients by using the DOTM algorithm, based on the PLS algorithm. The proposed strategy benefits from reducing the number of coefficients to be estimated in the DPD identification subsystem with respect to the non-dynamic conventional DPD estimation based on QR-LS.

\section{REFERENCES}

[1] J. Reina-Tosina, M. Allegue-Martinez, et al., "Behavioral modeling and predistortion of PAs under sparsity hypothesis," IEEE Trans. Microw. Theory Techn., vol. 63, no. 2, pp. 745-753, Feb. 2015.

[2] P. L. Gilabert, G. Montoro, et al., "Order reduction of wideband digital predistorters using PCA," in 2013 IEEE MTT-S International Microwave Symposium Digest (MTT). IEEE, jun 2013.

[3] R. Rosipal and N. Krämer, "Overview and recent advances in PLS," in Subspace, Latent Structure and Feature Selection. Springer Berlin Heidelberg, 2006, pp. 34-51.

[4] Q. A. Pham, D. López-Bueno, et al., "PLS identification of multiLUT digital predistorters for concurrent dual-band envelope tracking PAs," IEEE Trans. Microw. Theory Techn., pp. 1-8, 2018.

[5] S. de Jong, "SIMPLS: An alternative approach to PLS regression," Chemometrics and Intelligent Laboratory Systems, vol. 18, no. 3, pp. 251-263, Mar. 1993. 